\title{
Hybrid Stop Schedule of Urban Rail Train
}

\author{
Zheng-min Tan, Jie Cheng, Qi-yuan Peng, Mi Gan \\ College of Transportation \& Logistics, Southwest Jiaotong University (China) \\ zhengmintan@sina.com,cj1015@126.com,qivuan-peng@swjtu.edu.cn,migan@swjitu.cn
}

Received: November 2014

Accepted: March 2015

\section{Abstract:}

Purpose: In order to better serve the transport demand of urban area by rail, the Urban Rail Train Stop Schedule problem was considered.

Design/methodology/approach: Mathematic modeling as the Bi-level mathematical programming model with a game theory relation between the two level was used.

Findings: A 0-1 bi-level mathematical programming model for urban rail transit hybrid Stop Schedule is developed when game relation between train Stop Schedule and passenger transfer choice is considered.

Research limitations/implications: The research is formed under some assumption as the capacity is enough and the different trains are using separate rails.

Practical implications: ChongQing urban rail line 2 was taken as an example, the practical application of the model has proved the research can be feasible and efficiency in application.

Originality/value: A 0-1 bi-level mathematical programming model for urban rail transit hybrid Stop Schedule is developed which can schedule the train stops when considering better serve the demand. The upper level model is Stop Schedule targeting at the optimal profit from the operators side. The lower level model is passenger routing aims to minimize total travel time. The game relation of the two parts-operator and passenger were described by the 
interplay of the two level approach. According to its features, the bi-level model is integrated in order to be directly solvable by optimizing software.

Keywords: urban transit, urban rail, stop schedule, passenger routing, bi-level programming

\section{Introduction}

Public travel demand is greatly increased with the rapid development of urbanization within china. As an effective resolution of urban traffic and transportation problem, urban rail transit system has initialed a golden time of rapid development. Subsequently, the efficiency of urban rail operation has received more and more attention. Urban rail train operation problem has commonly been separated into several parts such as lining problem, frequency, service network and timetabling, in which train Stop Schedule for each train line is one of the basic components. The traditional way of train Stop Schedule (all-stop operation, standard Stop Schedule) can meet all the passenger demand easily. However some special Stop Schedule will better meet different passenger flow distribution when the effectiveness of operation is considered, they can be mainly classified into three kinds: skip-stop operation, zonal operation, express/local operation (Vuchic, 2004).

Worldwide research on Stop Schedule started earlier, many achievements can be found in 70ths and 80ths. Vuchic summarized types of Stop Schedule from characteristics, and then comparatively analyze and evaluate effectiveness for different Stop Schedule (Vuchic, 2004; Chang, Yeh \& Shen, 2000). Nevertheless, Worldwide application of special urban train stop is rare, such as New York, Moscow subway, light rail in San Francisco and Boston applied special stop plan in the peak period on some lines. It is probably because many cities do not meet the request of facility conditions for train to run special stop.

However, scholars become less interest in urban train stops and moved on to the lining and timetabling problem. What is more, in a related range, instead of continuing work on urban rail, lately scholars focus more on high speed railway or passenger dedicated line. Deng, Shi \& Zhou, (2009) analyzes the game relations between the stop setting and choice of passenger route, then established a bi-level model considering multi-class equilibrium passenger flow assignment as the lower level model, while the generalized travel cost minimum and stop quantity limitation as the objective function of the upper level model, the algorithm based on simulated annealing was designed (Deng, 2007; Deng, Shi \& Zhou, 2009). Although Stop Schedule of urban rail transit and high-speed railway both based on the passenger OD flow (Suh, Chon \& Rhee, 2007; Song, Xu \& Miao, 2006), there are still various difference between them according to physical conditions, operation subject and other environment aspects. Thus aiming at urban rail, GUO Yu set up a three 0-1 integer programming model for different Stop 
Schedule types combined with the passenger travel behavior when choosing from various routes (Guo, 2007). ZHENG Li established 0-1 integer programming model for skip-stop operation based on minimizing the general travel time of all passengers, tabu search is used to solve the problem (Zheng, Song, $\mathrm{He} \& \mathrm{Li}, 2009$ ). Then it refers to practice, all the urban rail inland china are still using the standard Stop Schedule. However, the varying passenger flow especially at peak hours require for some changes. Moreover a few cities are already equipped with triple or quadruple lines for special Stop Schedule at the planning horizon or even under construction.

In addition, many experts also included Stop Schedule into train lining problem or timetabling (Assis \& Milani, 2004; Fernández, 2010). However, urban rails are always operated individually by line and there are only one or two train routes for a single rail line, it mainly provides conditions to separate Stop Schedule from the whole operation pattern. And both from the passengers and operation aspects, Combined various types of Stop Schedule is better fitter the passenger flow with less equipment and save more time. Therefore, this paper aims at fixed out the optimal combined Stop Schedule for urban rail train based on the passengers' route choice.

\section{Problem Statement}

The train stop schedule is one of the important components in rail train operation not only in common rail system but also in urban railways. It is to decide if the train should stop or not at the stations along its route. From the operators' view, the less stop the less time consume thus less cost. however the passengers need more service at stations which request more stops. Much more similar to high speed rail, train stop setting follows the stackelberg game rules with passenger route choice, while train Stop Schedule is the leading participate and passengers are followers (DENG Lianbo, 2007; DENG Lianboet al., 2009). It is also the same case in urban rail transit and passenger routing.

Obviously, Stop Schedule and passenger routing are two interacted factors. On the one hand within the physical condition of railway lines and stations, train stop plan needs to meet the space-time distribution of passenger flow, while all the passenger flow route from beginning to the end point verify the spatial distribution of passenger flow. On the other hand, passengers choosing route from the train service network which provided by operation plans including train stop plans (Deng, 2007). Thus passenger flow shows a kind of collective behavior in a given transportation organization scheme, in other words passengers of the same OD will almost choose the same route. Therefore, different Stop Schedule would result different passenger flow routes. 
In addition, the train stop plan is made before the behavior choice, and constrains the scope and quality of passengers' choice, passenger routing which lead to the passenger flow distribution is the feedback to stop plan as well as other operation plans. So the Stop Schedule dominates passengers routing in this game. And the relationship between them can be described by the bi-level programming model, where the upper level is planning for stop plan, evaluating and adjusting according to passenger routing results, whereas the lower level is passenger routing on a given Stop Schedule, to realize the optimal set of passenger routes by choosing the least consuming ones.

In order to improve the efficiency of the service while at the same time guarantee the train stop service coverage, a set including two trains of the same path is taken as an units, named train A and B. Then from the aspects of integrated passengers and transport operation, Stop Schedule is made with input of dynamic passenger flow route.

\section{Upper Level Model}

\subsection{Conditions and Definitions}

The upper level mathematic model is Train Stop Schedule, which formed under such conditions:

1. Only one operation direction is considered.

2. The train running time will not be influenced by the Stop Schedule.

3. For an operation period of time, the same Stop Schedule is adopted.

4. For an operation period of time, the passenger flow uniformly distributed.

5. All passengers will get on the train they want.

6. Transfer time is independent of Stop Schedule.

Sets and indices:

S: Set of stations, $S=\{1,2, \ldots, n\}$, indexed by $i, s, o, d$.

T: Set of rail trains, which include two types of trains noted as $A$ and $B, T=\{A, B\}$.

$\mathrm{J}$ : Set of rail sections between stations, indexed by $\mathrm{j}$.

Decision variables:

$$
x_{A s}=\left\{\begin{array}{c}
1 \text { when A train stop at station s, } \\
0 \text { otherwise }
\end{array} \quad x_{B S}=\left\{\begin{array}{c}
1 \text { when B train stop at station s, } \\
0 \text { otherwise }
\end{array}\right.\right.
$$




\section{Parameters:}

total income: Number of railcars in block b.

total cost: Number of hazmat railcars in block b.

qod: Ticket price from station o to $d$.

pod: Passenger flow between station o and $d$.

C: Train operation cost per train kilometers.

NA: Number of train A needed in a planning horizon.

NB: Number of train B needed in a planning horizon.

L: Length of train route.

$\mathrm{I}_{1}$ : Time interval between train $\mathrm{A}$ and $\mathrm{B}$ at the first station.

$I_{2}$ : Time interval between train $B$ and $A$ at the first station.

$I_{\min }$ : The minimum time interval needed between train $B$ and $A$.

$e_{j}$ : Train running time in section $j$.

$h_{s}$ : Time spend each time train stop at station $\mathrm{s}$.

\section{Maximize}

$$
\text { total profit }=\text { total income }- \text { total cost }
$$

Subject to:

$$
\begin{gathered}
\text { total income }=\sum_{o, d} q_{o d} p_{o d} \\
\text { total cost }=C^{*}(N A+N B) * L \\
N A=T A /\left(I_{1}-I_{2}\right) \\
N B=T B /\left(I_{1}+I_{2}\right) \\
T A=\sum_{j} e_{j}+\sum_{s} x_{A s} h_{s} \\
T B=\sum_{j} e_{j}+\sum_{s} x_{B s} h_{s} \\
x_{A s}+x_{B s} \geq 1 \quad \forall s \in S \\
X_{A 1}=X_{A n}=X_{B 1}=X_{B n}=1
\end{gathered}
$$




$$
\begin{array}{cc}
\sum_{2}^{n-1} X_{A S} X_{B S} \geq 1 & \\
I_{1}+\sum_{1}^{i}\left(X_{A S}-X_{B S}\right) h_{S} \geq I_{\text {min }} & \forall i \in S \\
I_{2}+\sum_{1}^{i}\left(x_{B s}-x_{A s}\right) h_{s} \geq I_{\text {min }} & \forall i \in S \\
x_{A S} \in\{0,1\} & \forall s \in S \\
x_{B S} \in\{0,1\} & \forall s \in S
\end{array}
$$

The object function in (1) is formed from the view of the rail operators, based on maximizes total profit which equals to total income minus total cost.

The total income will exact denotes price income paying by all the passengers, that is showed in (2). The total cost estimates the train operation cost from (3) to (7). Constraints set (8) states that each OD pair of passenger flow can be served, in other words every station have at least one train served. All trains start from the car depot, complete the transportation task and return to the car depot, namely all trains stop at the first as well as the last station is represented by (9). (10) will ensure that transfer stations which have both kinds of trains served are not less than one. Constraints set (11) and (12) are track interval constraint, which is set in order to guarantee train departure time interval at any station is not less than tracking time interval. Finally, the sign restriction constraints are indicated in (13) and (14).

\section{Lower Level Model}

The train operation plan include Stop Schedule formed a service network for passenger traveling on it. In the service network, the passenger will choose the best route, which implied lowest consuming. When passengers choose the route, elements of many aspects will be considered which include fare, time, crowd, transit consumption etc., while travel time including transit time is the most direct and the decisive role of the influencing factors. Therefore, the lower level passenger routing model uses the minimal total travel time consuming as the goal. And passenger travel route is denoted by initial station, destination station, transfer station and the train to take on the sections. Then the model formed as follows: 
Decision variables:

$$
\begin{aligned}
& y_{A s}(o d)=\left\{\begin{array}{c}
1 \text { passenger flow of } o d \text { take train A and stop at station } s, \\
0 \text { otherwise }
\end{array}\right. \\
& y_{B S}(o d)=\left\{\begin{array}{c}
1 \text { passenger flow of } o d \text { take train B and stop at station } s, \\
0 \text { otherwise }
\end{array}\right.
\end{aligned}
$$

\section{Parameters:}

total travel time: Time consuming for all the passengers.

$\mathrm{u}_{\mathrm{o}}: \quad$ Passenger waiting time to board at station $\mathrm{o}$.

\section{Minimize}

$$
\text { total travel time }=\sum_{o, d} p_{o d}\left(u_{o}+\sum_{1}^{n}\left(y_{A s}(o d)+y_{B s}(o d)\right) h_{s}+\sum_{1}^{n}\left(y_{A s}(o d) y_{B s}(o d)\right) r_{s}\right)
$$

Subject to:

$$
\begin{aligned}
& u_{o}=\left(I_{1}+\sum_{1}^{o}\left(x_{A s}-x_{B s}\right) h_{s}+I_{2}+\sum_{1}^{o}\left(x_{B s}-x_{A s}\right) h_{s}\right. \\
& \sum_{1}^{n} y_{A s}(o d)+\sum_{1}^{n} y_{B s}(o d) \geq 1 \quad s \in[o, d] \quad o, d \in S \\
& y_{A S}(o d) \leq x_{A s} \quad s \in[o, d] \quad o, d \in S \\
& y_{B s}(o d) \leq x_{B s} \quad s \in[o, d] \quad o, d \in S \\
& y_{A s}(o d) y_{B s}(o d) \leq x_{A s} x_{B s} \quad s \in[o, d] \quad o, d \in S \\
& y_{A d}(o d) y_{A d}(o d)+y_{B o}(o d) y_{B d}(o d)+\sum_{o}^{d} y_{A s}(o d) y_{B s}(o d) \leq 1 \quad o, d \in S \\
& y_{A S}(o d) \in\{0,1\} \quad \forall s \in S \\
& y_{A S}(\text { od }) \in\{0,1\} \quad \forall s \in S
\end{aligned}
$$

As we take the total travel time consuming as the goal in (15), an factor should be considered that the section running time could be eliminate while it will not vary with stop schedule. (16) shows the way to calculate passenger waiting time under the condition that passengers are uniformly arrive the stations, that the expected waiting time is half of the interval. Constraints set (17) states that passenger route should be continuous from the start to the end. (18), (19) 
will ensure that passengers can only choose on board to the train stop at that station. Then transfer station will be served by both two kinds of trains in constrains (20). (21) means passengers can only travel from $O$ to $D$ without return, in another way expressed as passengers either take one train for $O$ to $D$ or can only transfer at the station between $O$ and D. Finally, the sign restriction constraints are indicated in (22) and (23).

\section{Model Integration and Solving}

Bi-level programming model is always treated in different ways. For the best of our knowledge, there is a lack of mature and effective method to solve Bi-level model. The optimal solution is always on one of the level while the other level may not get the best solution when being constrained in solution space.

However, when it comes to urban rail, there are particularly reasons to integrate the two level models into one. First of all, the urban rail operator is always financially supported by the local government, its particular features requires more public service point of view, which means besides profit, the operator also have the mission to better service the passengers by maximizing the their interest. In this way, it consists with the lower level planning goal. Accordingly, the objective functions and constraints of the two levels are respectively integrated in order to find solving method, thus end up with a multi-objective 0-1 nonlinear programming model.

After integration the model is as follows:

\section{Minimize}

$$
Z=-\gamma_{1} * \text { total profit }+\gamma_{2} * \text { total travel time }
$$

Subject to:

For the multi-objective, a weighted coefficient method is used in (24), each objective function multiplied by the weight coefficient- $\mathrm{\gamma} 1$ and $\mathrm{y} 2$.

It is not hard to observe from the above model that number of OD pairs is the important controlling infector of the model size, if the number of OD pairs can be reduced, then it will effectively reduce the total number of decision variables and constraints. After integration the model can be optimal solved by existing software such as LINGO11.0 programming solution. 


\section{Case Study}

\subsection{Case \# 1: Six Nodes}

In this case, there are six stations along the train route, denoted by $1-6$. Train stop for 1 min at each station, the minimum train tracking interval Imin $=2 \mathrm{~min}$, the station departure interval is $5 \mathrm{~min}$, ticket price is 2 yuan, train cost 50 yuan/ $\mathrm{km}$, train route is $20 \mathrm{~km}$ long. The OD passenger flows are shown in Table 1.

\begin{tabular}{|c|c|c|c|c|c|c|}
\hline Station & $\mathbf{1}$ & $\mathbf{2}$ & $\mathbf{3}$ & $\mathbf{4}$ & $\mathbf{5}$ & $\mathbf{6}$ \\
\hline 1 & 0 & 50 & 50 & 50 & 50 & 50 \\
\hline 2 & 0 & 0 & 50 & 50 & 50 & 50 \\
\hline 3 & 0 & 0 & 0 & 50 & 50 & 50 \\
\hline 4 & 0 & 0 & 0 & 0 & 50 & 50 \\
\hline 5 & 0 & 0 & 0 & 0 & 0 & 50 \\
\hline 6 & 0 & 0 & 0 & 0 & 0 & 0 \\
\hline
\end{tabular}

Table 1. OD passenger flows of case \#1

\section{Basic Scenario:}

In the basic scenario, weight coefficients of the objective function are given as 0.5 and 0.5 respectively. Programmed in mathematical optimization software LINGO11.0, the solving process took 2 minutes 11 seconds on a computer of CPU $2.13 \mathrm{G}$, memory $1 \mathrm{~GB}$, the results are as follows:

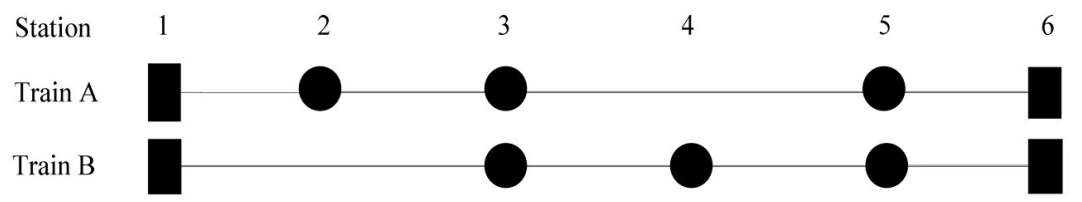

Figure 1. Train stop schedule for basic sinario in case \#1

\section{Sinario 2:}

The sensitivity of the weight coefficients of the objective function is analyzed in this sinario, by taking different weight coefficients of the objective function from 0 to 1 . The results of LINGO 11.0 solution are shown in Table 2 and Figure 2: 


\begin{tabular}{|c|c|c|c|c|c|}
\hline$(\mathbf{Y} \mathbf{1}, \mathbf{Y})$ & $\mathbf{A}$ & $\mathbf{B}$ & $\mathbf{Z}$ & total profit & Total traveltime \\
\hline$(1,0)$ & 123456 & 123456 & 2250 & 2250 & 3000 \\
\hline$(0.9,0.1)$ & 123456 & 123456 & 2325 & 2250 & 3000 \\
\hline$(0.8,0.2)$ & 12356 & 13456 & 2380 & 2350 & 2500 \\
\hline$(0.7,0.3)$ & 12356 & 13456 & 2395 & 2350 & 2500 \\
\hline$(0.6,0.4)$ & 12356 & 13456 & 2410 & 2350 & 2500 \\
\hline$(0.5,0.5)$ & 12356 & 13456 & 2425 & 2350 & 2500 \\
\hline$(0.4,0.6)$ & 12356 & 13456 & 2440 & 2350 & 2500 \\
\hline$(0.3,0.7)$ & 12356 & 13456 & 2455 & 2350 & 2500 \\
\hline$(0.2,0.8)$ & 1456 & 12346 & 2402 & 3012 & 2250 \\
\hline$(0.1,0.9)$ & 1456 & 12346 & 2326 & 3012 & 2250 \\
\hline$(0,1)$ & 123456 & 126 & 2250 & 4437 & 2250 \\
\hline
\end{tabular}

Table 2. Weight coefficient sensitivity analysis

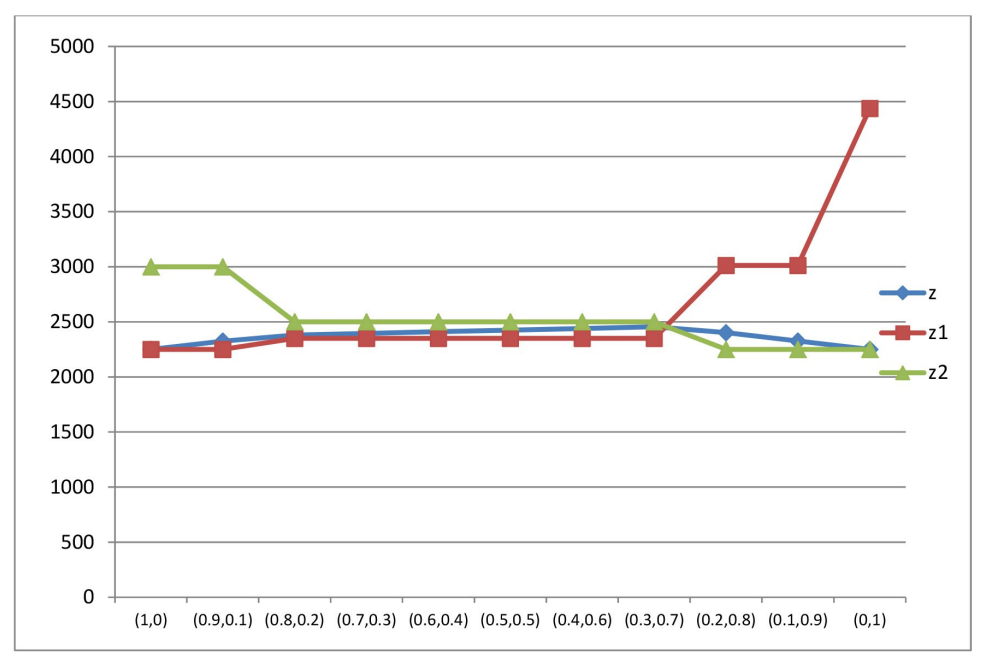

Figure 2. Weight coefficient sensitivity analysis

The table and figure shows that first, there is an obvious robustness for the experiment result. When the weight coefficient vary from $(0.8,0.2)$ to $(0.3,0.7)$, the optimal solution and the objective function optimal value are stable.

Second, when objective one is the major target, namely the weight coefficient $(1,0)$ and $(0.9,0.9)$, the optimal solutions is the standard stop plan which stop at each station. Visible in the case of passenger flow distribution more uniform, standard of stops is to satisfy all passengers travel which cost the shortest time.

The third, as the operating profit become more and more important, the optimal scheme has changed from $Y_{2}=0.8$. The result showed that the total number of stops is lessening while lower operating costs performed. But at the same time it will reduce the train service frequency at the station, all passengers total travel time consumption increase. 
Finally, the two objective functions comes from two opposite directions, one of the optimization is always at the expense of deteriorate another. Because passenger welfare is the common interests of both operating enterprise and passengers, it need more priority compared to the profit target that considered only operating companies.

\subsection{Case \# 2: Real Case}

The Chongqing rail transit line 2 is straight light rail of total 18 stations. The up direction nonzero OD pair of passenger demand is $C_{18}^{2}=153$. Stop time $1 \mathrm{~min}$, the minimum tracking time interval 2 min. Predicted passenger demand of 2015 is taken.

The objective function weight coefficients were 0.5 and 0.5. 25 minutes 29 seconds were taken to calculate. The results are as follows:

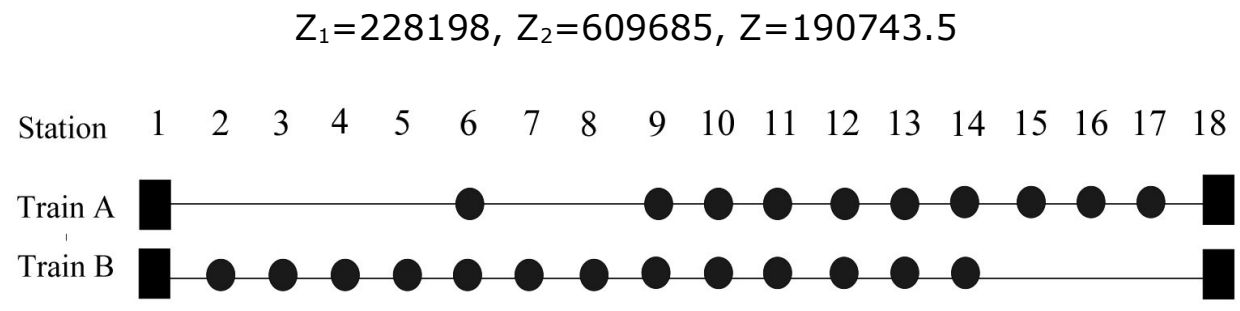

Figure 3. Train Stop Schedule for case \#2

It is fair to conclude that the optimal model is feasible under a given parameter condition.

On one hand, compared to standard stop scheme, this combined stop schedule decrease 4.5 stop frequency for each train. It reduced all passengers total travel time 314413 mins. On the other hand, for operation aspects, it saved 4.5 min per running cycle, hence reduce vehicle usage and cut down cost.

However, the service frequency of intermediate stations is limited, which result in longer waiting time thus it is more appropriate to applied when train interval is quite small. What is more, as train $A$ and $B$ not service all the stations, transfer is required for some $O D$ passengers.

\section{Conclusions}

In this paper, the hybrid train Stop Schedule of urban rail transit based on passenger route choice was studied and optimized from multiple viewpoint, which will effectively reduce the operation cost in the mean while improve the urban rail transit system in service level. 
1. A mathematical model to optimize combined train Stop Schedule of urban rail transit was established and solving method is given which can schedule the train stops when considering better serve the demand.

2. Analysis the game relation between Stop Schedule and passenger routing, then bi-level programming was applied to include the influence of passenger behavior on Stop Schedule. The game relation of the two parts-operator and passenger were described by the interplay of the two level approach.

3. When common ground was seek while differences were put aside, the final set of Stop Schedule will satisfy various needs by targeting at the least general passenger travel time.

4. Through the case study and illustrates, the feasibility and validity of the model was proved, it also explained that special Stop Schedule can be carried out under some conditions of passenger flow, like the major density in peak hours.

5. Although there are only two trains in a unit group as a object was studied in this paper, the model itself will not be restrict that way, it can be easily extended to solving more kinds of trains.

However, affecting factors like the random characteristics of arrival, capacity of line and train as well as the most suitable weight coefficient still require for more research. In addition, special Stop Schedule may result in different train speeds, which will lead to new transport organization problems, such as the more lines at station for train to pass over (like New York City). What is more accurate train stop information service should be provided for passengers to choose the best route.

\section{Acknowledgement}

This work is partially supported by the National Nature Science Foundation (No. 60776827) in China, the Fundamental Research Funds for the Central Universities (Project No. 10501B10096017), the People's Republic of China; Southwest Jiaotong University Emei Branch Special funds for high level talents (Project No. 10501X10096015).

\section{References}

Assis, W.O., \& Milani, B.E. (2004). Generation of optimal schedules for metro lines using model predictive control. Automatica Mag, 40, 1397-1404. 
Chang, Y.H., Yeh, C.H., \& Shen, C.C. (2000). A multi-objective model for passenger train services planning application to Taiwan's high-speed rail line. Transportation Research Part $B$ Mag, 34, 91-106. http://dx.doi.org/10.1016/S0191-2615(99)00013-2

Deng, L. (2007). Study on the Optimal Problems of Passenger Train Plan for Dedicated Passenger Traffic Line. Wuhan: Central South University, 76-85.

Deng, L., Shi, F., \& Zhou, W. (2009). Stop Schedule Plan Optimization for Passenger Train. China Railway Science Mag, 30, 103-107.

Fernández, R. (2010). Modeling public transport stops by microscopic simulation. Transportation Research Part C Mag, 18, 856-868. http://dx.doi.org/10.1016/j.trc.2010.02.002

Guo, Y. (2007). Study on Optimal Problems of Stop Schedule Plan for Urban Rail Transit. Beijing: Beijing Jiaotong University, 30-47.

Song, J., Xu, R., \& Miao, H. (2006). Problems of Operating the Express/Slow Train on the Regional Urban Rail Transit Line. Urban Mass Transit Mag, 12, 23-37.

Suh, W., Chon, K.S. \& Rhee. S.M. (2007). Effect of Skip-Stop Policy on a Korean Subway System. Transportation Research Record: Journal of the Transportation Research Board Mag, 1793, 33-39. http://dx.doi.org/10.3141/1793-05

Vuchic, V.R. (2004). Urban Transit: Operation, Planning and Economics. New Jersey: John Wiley \& Sons, Inc, 44-51.

Zheng, L., Song, R., He, S.W., \& Li, H.D. (2009). Optimization Model and Algorithm of Skipstop Strategy for Urban Rail Transit. Journal of the China Railway Society. Mag, 31, 1-8.

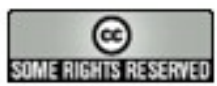

Article's contents are provided on a Attribution-Non Commercial 3.0 Creative commons license. Readers are allowed to copy, distribute and communicate article's contents, provided the author's and Journal of Industrial Engineering and Management's names are included. It must not be used for commercial purposes. To see the complete license contents, please visit http://creativecommons.org/licenses/by-nc/3.0/. 